\title{
Bounds on normalized Laplacian eigenvalues of graphs
}

\author{
Jianxi Li $i^{1,2^{*}}$, Ji-Ming Guo ${ }^{3}$ and Wai Chee Shiu ${ }^{4}$
}

${ }^{*}$ Correspondence: ptjxli@hotmail.com

'School of Mathematics and Statistics, Minnan Normal University, Zhangzhou, Fujian, P.R. China ${ }^{2}$ Center for Discrete Mathematics, Fuzhou University, Fuzhou, Fujian, P.R. China

Full list of author information is available at the end of the article

\begin{abstract}
Let $G$ be a simple connected graph of order $n$, where $n \geq 2$. Its normalized Laplacian eigenvalues are $0=\lambda_{1} \leq \lambda_{2} \leq \cdots \leq \lambda_{n} \leq 2$. In this paper, some new upper and lower bounds on $\lambda_{n}$ are obtained, respectively. Moreover, connected graphs with $\lambda_{2}=1$ (or $\left.\lambda_{n-1}=1\right)$ are also characterized.
\end{abstract}

MSC: 05C50; $15 \mathrm{~A} 48$

Keywords: normalized Laplacian eigenvalue; largest eigenvalue; bound

\section{Introduction}

Let $G$ be a graph with vertex set $V(G)$ and edge set $E(G)$. Its order is $|V(G)|$, denoted by $n$, and its size is $|E(G)|$, denoted by $m$. In this paper, all graphs are simple connected of order $n \geq 2$. For $v \in V(G)$, let $d(v)$ and $N(v)$ be the degree and the set of neighbors of $v$, respectively. The maximum and minimum degrees of $G$ are denoted by $\Delta$ and $\delta$, respectively.

Let $A(G)$ and $D(G)$ be the adjacency matrix and the diagonal matrix of vertex degrees of $G$, respectively. The Laplacian and normalized Laplacian matrices of $G$ are defined as $L(G)=D(G)-A(G)$ and $\mathcal{L}(G)=D(G)^{-1 / 2} L(G) D(G)^{-1 / 2}$, respectively. When only one graph $G$ is under consideration, we sometimes use $A, D, L$ and $\mathcal{L}$ instead of $A(G), D(G), L(G)$ and $\mathcal{L}(G)$, respectively. It is easy to see that $\mathcal{L}(G)$ is a symmetric positive semidefinite matrix and $D(G)^{1 / 2} \mathbf{1}$ is an eigenvector of $\mathcal{L}(G)$ with eigenvalue 0 , where $\mathbf{1}$ is the vector with all ones. Thus, the eigenvalues $\lambda_{i}(G)(1 \leq i \leq n)$ of $\mathcal{L}(G)$ (or the normalized Laplacian eigenvalues of $G$ ) satisfy

$$
\lambda_{n}(G) \geq \cdots \geq \lambda_{2}(G) \geq \lambda_{1}(G)=0 .
$$

Some of them may be repeated according to their multiplicities. We call $\lambda_{k}(G)$ the $k$ th smallest normalized Laplacian eigenvalue of $G$. When only one graph $G$ is under consideration, we sometimes write $\lambda_{k}$ instead of $\lambda_{k}(G)$, for $1 \leq k \leq n$.

The normalized Laplacian is mentioned briefly in the recent monograph by Cvetković et al. [1]; however, the standard reference for it is the monograph by Chung [2], which deals almost entirely with this matrix. The normalized Laplacian eigenvalues can be used to give useful information about a graph [2]. For example, one can obtain the number of connected components from the multiplicity of the eigenvalue 0 , the bipartiteness from its $\lambda_{n}$ (which is at most 2), as well as the connectivity from its $\lambda_{2}$. Moreover, $\lambda_{2}$ is also

@2014 Li et al.; licensee Springer. This is an Open Access article distributed under the terms of the Creative Commons Attribution License (http://creativecommons.org/licenses/by/2.0), which permits unrestricted use, distribution, and reproduction in any medium, provided the original work is properly cited. 
closely related to the discrete Cheeger's constant, isoperimetric problems, etc. (see [2]). Chen and Jost [3] established the relationship between minimum vertex covers and the eigenvalues of the normalized Laplacian on trees. Some upper bounds for $\lambda_{n}$ have been introduced by Rojo and Soto [4] and Banerjee [5], respectively. For more results on the normalized Laplacian eigenvalues of graphs can be found in $[2,6,7]$.

In this paper, some new upper and lower bounds on $\lambda_{n}$ of a graph in terms of its maximum degree, covering number etc., are deduced, respectively. Moreover, connected graphs with $\lambda_{2}=1$ (or $\lambda_{n-1}=1$ ) are also characterized.

\section{Preliminaries}

Here we recall some basic properties of the eigenvalues and eigenfunctions of the normalized Laplacian matrix of a graph $G$.

Let $\mathbf{g}: V(G) \rightarrow \mathbb{R}^{n}$ which assigns to each vertex $v$ of $G$ a real value $g(v)$, the coordinate of $\mathbf{g}$ according to $v$. Let $\mathbf{f}=D^{-1 / 2} \mathbf{g}$. Then we have

$$
\frac{\mathbf{g}^{T} \mathcal{L} \mathbf{g}}{\mathbf{g}^{T} \mathbf{g}}=\frac{\mathbf{f}^{T} D^{1 / 2} \mathcal{L} D^{1 / 2} \mathbf{f}}{\left(D^{1 / 2} \mathbf{f}\right)^{T} D^{1 / 2} \mathbf{f}}=\frac{\mathbf{f}^{T} L \mathbf{f}}{\mathbf{f}^{T} D \mathbf{f}}=\frac{\sum_{u v \in E(G)}(f(u)-f(v))^{2}}{\sum_{v \in V(G)} d(v) f(v)^{2}}
$$

Thus, the following formula for $\lambda_{n}$ is clear:

$$
\lambda_{n}=\sup _{\mathbf{f} \perp D \mathbf{1}} \frac{\sum_{u v \in E(G)}(f(u)-f(v))^{2}}{\sum_{v \in V(G)} d(v) f(v)^{2}} .
$$

A vector $\mathbf{f}$ that satisfies equality in Eq. (2.1) is called a harmonic eigenfunction of $\mathcal{L}$ associated with $\lambda_{n}(G)$.

Proposition 2.1 ([2]) Let $G$ be a graph and $\mathbf{f}$ be a harmonic eigenfunction of $\mathcal{L}$ associated with $\lambda_{n}(G)$. Then for any $v \in V(G)$, we have

$$
f(v)-\frac{1}{d(v)} \sum_{u v \in E(G)} f(u)=\lambda_{n}(G) f(v) .
$$

\section{Main result}

We call $G$ a triangulation, if every pair of adjacent vertices of $G$ have at least one common adjacent vertex. A planar graph is called a maximal planar graph if for every pair of nonadjacent vertices $u$ and $v$ of $G$, the graph $G+u v$ is nonplanar. Lu et al. [8] and Guo et al. [9] gave the upper bounds for the Laplacian spectral radius of a triangulation and a maximal planar graph, respectively. For the normalized Laplacian spectral radius, we have the following somewhat similar result.

Theorem 3.1 Let $G=(V, E)$ be a triangulation of order $n$. Then

$$
\lambda_{n} \leq \max \left\{\frac{2 d\left(v_{i}\right)-1+\sqrt{4 d\left(v_{i}\right) m\left(v_{i}\right)-4 d\left(v_{i}\right)+1}}{2 d\left(v_{i}\right)}: v_{i} \in V\right\}
$$

where $m\left(v_{i}\right)=\sum_{v_{j} \in N\left(v_{i}\right)} d\left(v_{j}\right) / d\left(v_{i}\right)$ is the average 2-degree of the vertex $v_{i}$. Moreover, the equality holds if $G \cong K_{3}$. 
Proof Let $V=\left\{v_{1}, \ldots, v_{n}\right\}$, and let $\mathbf{f}=\left(f\left(v_{1}\right), \ldots, f\left(v_{n}\right)\right)^{T}$ be the harmonic eigenfunction of $\mathcal{L}(G)$ corresponding to $\lambda_{n}$. Then by Proposition 2.1, we have for each $v_{i} \in V$,

$$
d\left(v_{i}\right)\left(1-\lambda_{n}\right) f\left(v_{i}\right)=\sum_{v_{i} v_{j} \in E} f\left(v_{j}\right)
$$

Hence by the Lagrange identity, we have for each $v_{i}$,

$$
d\left(v_{i}\right)^{2}\left(1-\lambda_{n}\right)^{2} f\left(v_{i}\right)^{2}=d\left(v_{i}\right) \sum_{v_{i} v_{j} \in E} f\left(v_{j}\right)^{2}-\sum_{\substack{1 \leq j<k \leq n \\ v_{j}, v_{k} \in N\left(v_{i}\right)}}\left(f\left(v_{j}\right)-f\left(v_{k}\right)\right)^{2} .
$$

Sum over $v_{i}$ to obtain

$$
\begin{aligned}
& \sum_{i=1}^{n} d\left(v_{i}\right)^{2}\left(1-\lambda_{n}\right)^{2} f\left(v_{i}\right)^{2} \\
& =\sum_{i=1}^{n} d\left(v_{i}\right) \sum_{v_{i} v_{j} \in E} f\left(v_{j}\right)^{2}-\sum_{i=1}^{n} \sum_{\substack{1 \leq j<k \leq n \\
v_{j}, v_{k} \in N\left(v_{i}\right)}}\left(f\left(v_{j}\right)-f\left(v_{k}\right)\right)^{2} \\
& =\sum_{i=1}^{n} d\left(v_{i}\right) m\left(v_{i}\right) f\left(v_{i}\right)^{2}-\sum_{i=1}^{n} \sum_{\substack{1 \leq j<k \leq n \\
v_{j}, v_{k} \in N\left(v_{i}\right)}}\left(f\left(v_{j}\right)-f\left(v_{k}\right)\right)^{2},
\end{aligned}
$$

where $m\left(v_{i}\right)=\sum_{v_{j} \in N\left(v_{i}\right)} d\left(v_{j}\right) / d\left(v_{i}\right)$.

Note that $G$ is a triangulation. Then by Eq. (2.1), we have

$$
\sum_{i=1}^{n} \sum_{\substack{1 \leq j<k \leq n \\ v_{j}, v_{k} \in N\left(v_{i}\right)}}\left(f\left(v_{j}\right)-f\left(v_{k}\right)\right)^{2} \geq \sum_{\substack{1 \leq j<k \leq n \\ v_{j} v_{k} \in E(G)}}\left(f\left(v_{j}\right)-f\left(v_{k}\right)\right)^{2}=\lambda_{n} \sum_{i=1}^{n} d\left(v_{i}\right) f\left(v_{i}\right)^{2}
$$

Thus, combining Eqs. (3.1) and (3.2), we have

$$
\sum_{i=1}^{n}\left[d\left(v_{i}\right)^{2}\left(1-\lambda_{n}\right)^{2}-d\left(v_{i}\right) m\left(v_{i}\right)+\lambda_{n} d\left(v_{i}\right)\right] f\left(v_{i}\right)^{2} \leq 0
$$

This implies that there exists at least one vertex $v_{i}$ such that

$$
d\left(v_{i}\right)^{2}\left(1-\lambda_{n}\right)^{2}-d\left(v_{i}\right) m\left(v_{i}\right)+\lambda_{n} d\left(v_{i}\right) \leq 0 .
$$

That is,

$$
\lambda_{n} \leq \max \left\{\frac{2 d\left(v_{i}\right)-1+\sqrt{4 d\left(v_{i}\right) m\left(v_{i}\right)-4 d\left(v_{i}\right)+1}}{2 d\left(v_{i}\right)}: v_{i} \in V\right\} .
$$

For $G=K_{3}$, it is easy to check that the equality holds.

Furthermore, we have the following more general result. 
Theorem 3.2 Let $G=(V, E)$ be a simple connected graph of order $n$ with $m$ edges. If each edge of $G$ belongs to at least $t$ triangles $(t \geq 1)$, then

$$
\lambda_{n} \leq \max \left\{\frac{2 d\left(v_{i}\right)-t+\sqrt{4 d\left(v_{i}\right) m\left(v_{i}\right)-4 t d\left(v_{i}\right)+t^{2}}}{2 d\left(v_{i}\right)}: v_{i} \in V\right\},
$$

the equality occurs if $G$ is the complete graph $K_{t+2}$.

Proof For $G=K_{t+2}$, it is easy to check that the equality in Eq. (3.3) holds. If we replace Eq. (3.2) in the proof of Theorem 3.1 by

$$
\sum_{i=1}^{n} \sum_{\substack{1 \leq j<k \leq n \\ v_{j}, v_{k} \in N\left(v_{i}\right)}}\left(f\left(v_{j}\right)-f\left(v_{k}\right)\right)^{2} \geq t \sum_{\substack{1 \leq j<k \leq n \\ v_{j} v_{k} \in E}}\left(f\left(v_{j}\right)-f\left(v_{k}\right)\right)^{2}=t \lambda_{n} \sum_{i=1}^{n} d\left(v_{i}\right) f\left(v_{i}\right)^{2},
$$

then the result follows.

For the maximal planar graphs, we have the following upper bound.

Theorem 3.3 Let $G$ be a maximal planar graph of order $n \geq 4$ with $m$ edges. Then

$$
\lambda_{n} \leq \max \left\{\frac{d\left(v_{i}\right)-1+\sqrt{d\left(v_{i}\right) m\left(v_{i}\right)-2 d\left(v_{i}\right)+1}}{d\left(v_{i}\right)}: v_{i} \in V(G)\right\} .
$$

Proof Note that for any maximal planar graph $G$, each edge of $G$ belongs to at least 2 triangles. Then the result follows from Theorem 3.2.

In what follows, we turn to some lower bounds on $\lambda_{n}$. The following result due to Chung [2] concerns the lower bound on $\lambda_{n}(G)$.

Lemma 3.4 ([2]) Let $G$ be a connected graph of order $n$. Then $\lambda_{n}(G) \geq \frac{n}{n-1}$, the equality holds if and only if $G \cong K_{n}$, where $K_{n}$ is the complete graph of order $n$.

Let $G=(V, E)$ be a graph and $X \subseteq V$ be a subset of the vertices. Let $\bar{X}=V \backslash X$ be the complement of the set $X$. The volume of $X$ is defined to be the sum of the degrees of the vertices in $G$, that is,

$$
\operatorname{vol}(X)=\sum_{v \in X} d(v)
$$

Note that $\operatorname{vol}(V)$ is equal to twice the number of edges in the graph.

Theorem 3.5 Let $G$ be a connected graph of order $n$ with $m$ edges. For any nonempty subset $X \subseteq V$, we have

$$
\lambda_{n} \geq \frac{2 m\left|E_{X}\right|}{\operatorname{vol}(X)(2 m-\operatorname{vol}(X))},
$$

where $E_{X}$ is the set of all edges with one end in $X$ and the other end in $\bar{X}$. Moreover, if the equality holds, then $\frac{\sum_{u \in N(v)} f(u)}{d(v)}=x$ for each $v \in X$ and $\frac{\sum_{u \in N(v)} f(u)}{d(v)}=y$ for each $v \in \bar{X}$, where $x$ and $y$ are constant such that $\frac{x}{y}=-\frac{\operatorname{vol}(\bar{X})}{\operatorname{vol}(X)}$. 
Proof Let $X \subseteq V$ and $\mathbf{f}$ be a vector such that

$$
f(u)= \begin{cases}-\operatorname{vol}(\bar{X}) & \text { if } u \in X \\ \operatorname{vol}(X) & \text { if } u \notin X\end{cases}
$$

Clearly, $\sum_{u \in V} d(u) f(u)=-\operatorname{vol}(\bar{X}) \operatorname{vol}(X)+\operatorname{vol}(\bar{X}) \operatorname{vol}(X)=0$. Moreover, note that $\operatorname{vol}(X)+$ $\operatorname{vol}(\bar{X})=\operatorname{vol}(V)=2 m$. Then, by Eq. (2.1), we have

$$
\begin{aligned}
\lambda_{n} & \geq \frac{\sum_{u v \in E(G)}(f(u)-f(v))^{2}}{\sum_{v \in V(G)} d(v) f(v)^{2}} \\
& =\frac{\left|E_{X}\right|(\operatorname{vol}(X)+\operatorname{vol}(\bar{X}))^{2}}{\operatorname{vol}(X) \operatorname{vol}(\bar{X})(\operatorname{vol}(X)+\operatorname{vol}(\bar{X}))} \\
& =\frac{2 m\left|E_{X}\right|}{\operatorname{vol}(X)(2 m-\operatorname{vol}(X))} .
\end{aligned}
$$

Moreover, if the equality holds, then $\mathbf{f}$ is the harmonic eigenfunction of $\mathcal{L}$ associated with $\lambda_{n}(G)$. Hence Proposition 2.1 implies that

$$
\begin{cases}\left(\lambda_{n}-1\right) \operatorname{vol}(\bar{X})=\frac{\sum_{u \in N(v)} f(u)}{d(v)} & \text { for each } v \in X \\ \left(1-\lambda_{n}\right) \operatorname{vol}(X)=\frac{\sum_{u \in N(v)} f(u)}{d(v)} & \text { for each } v \in \bar{X}\end{cases}
$$

Let $x=\left(\lambda_{n}-1\right) \operatorname{vol}(\bar{X})$ and $y=\left(1-\lambda_{n}\right) \operatorname{vol}(X)$. Then $\frac{x}{y}=-\frac{\operatorname{vol}(\bar{X})}{\operatorname{vol}(X)}$. This completes the proof.

Let $X=\{u\}$ in Theorem 3.5. Note that $\operatorname{vol}(X)=d(u)=\left|E_{X}\right|$. Then we have the following.

Corollary 3.6 Let $G$ be a graph of order $n$ with $m$ edges. Then

$$
\lambda_{n} \geq \frac{2 m}{2 m-\Delta}
$$

where $\Delta$ is the maximum degree of $G$.

Remark 3.1 Note that $2 m \leq n \Delta$ holds for any graph of order $n$ with $m$ edges and maximum degree $\Delta$. Thus the lower bound in Corollary 3.6 is always better than that in Lemma 3.4. Moreover, if $G$ is a complete graph $K_{n}$ or a star $S_{n}$, then it is easy to check that the equality holds in Eq. (3.5).

Similarly, let $X=\{u, v\}$ in Theorem 3.5. Then we have:

Corollary 3.7 Let $G$ be a graph of order $n$ with $m$ edges. Let $a=\max _{u v \in E(G)}\{d(u)+d(v)\}$ and $b=\max _{u v \notin E(G)}\{d(u)+d(v)\}$. Then:

(1) $\lambda_{n} \geq \frac{2 m(a-2)}{a(2 m-a)}$, and the equality holds if $G \cong K_{n}$.

(2) $\lambda_{n} \geq \frac{2 m}{2 m-b}$, and the equality holds if $G \cong K_{2, n-2}$, where $G \cong K_{2, n-2}$ is the complete bipartite graph with parts of cardinalities 2 and $n-2$. 
Proof Let $X=\{u, v\}$ in Theorem 3.5. If $u v \in E(G)$, then $\left|E_{X}\right|=d(u)+d(v)-2$ and $\operatorname{vol}(X)=$ $d(u)+d(v)$. Theorem 3.5 implies that

$$
\lambda_{n} \geq \frac{2 m[(d(u)+d(v))-2]}{(d(u)+d(v))[2 m-(d(u)+d(v))]} .
$$

Let $f(x)=\frac{2 m(x-2)}{x(2 m-x)}$ for $x>2$. Then it is easy to see that $f(x)$ is increasing on $x$. Hence, we have $\lambda_{n} \geq \frac{2 m(a-2)}{a(2 m-a)}$. Moreover, it is easy to check that the equality holds when $G \cong K_{n}$.

If $u v \notin E(G)$, then $\left|E_{X}\right|=\operatorname{vol}(X)=d(u)+d(v)$. Theorem 3.5 implies that

$$
\lambda_{n} \geq \frac{2 m}{2 m-(d(u)+d(v))} .
$$

Hence $\lambda_{n} \geq \frac{2 m}{2 m-b}$. Moreover, it is easy to check that the equality holds when $G \cong K_{2, n-2}$.

A set of vertices $X$ of $G$ is called a cover of $G$ if every edge of $G$ is incident to some vertex in $X$. The least cardinality of a cover of $G$ is called the covering number of $G$ and denoted by $\tau(G)$. It is clear that if a vertex set $X$ is a vertex cover if and only if $\bar{X}$ is an independent set. The following lower bound for $\lambda_{n}$ in terms of $\tau(G)$ is obtained.

Theorem 3.8 Let $\mathrm{G}$ be a graph order $n$ with $m$ edges. Then

$$
\lambda_{n} \geq \frac{2 m}{2 m-\delta(n-\tau(G))},
$$

where $\delta$ is the minimum degree of $G$. Moreover, the equality holds if $G \cong C_{n}$ when $n$ is even, $G \cong K_{a, b}$ or $G \cong K_{n}$, where $C_{n}$ is the cycle of order $n$ and $K_{a, b}$ is the complete bipartite graph with parts of cardinalities $a$ and $b$.

Proof Let $X$ be a minimal covering set of $G$ with $|X|=\tau(G)$. Then $\bar{X}$ is an independent set. Hence $\operatorname{vol}(\bar{X})=\left|E_{X}\right|$ and $\operatorname{vol}(X)=2 m-\left|E_{X}\right|$. Then Theorem 3.5 implies that $\lambda_{n} \geq \frac{2 m}{2 m-\left|E_{X}\right|}$. Moreover, by the definition of covering set, we have $\left|E_{X}\right| \geq \delta(n-\tau(G))$. Hence we have $\lambda_{n} \geq \frac{2 m}{2 m-\delta(n-\tau(G))}$. Moreover, if $G \cong C_{n}$ when $n$ is even, then $\tau(G)=\frac{n}{2}$. Hence it is easy to check that the equality holds. Similarly, if $G \cong K_{a, b}$ or $G \cong K_{n}$, then the equality holds. This completes the proof.

Chung [2] proved that for any graph $G$ of order $n, \lambda_{2} \leq \frac{n}{n-1}$ with equality holding if and only if $G \cong K_{n}$. Moreover, the following result is also introduced.

Lemma 3.9 ([2]) Let $G\left(G \neq K_{n}\right)$ be a connected graph of order $n$. Then $\lambda_{2} \leq 1$.

In what follows, we characterize all connected graphs with $\lambda_{2}=1$. We will make use of the following lemma.

Lemma 3.10 ([7]) Let $G$ be a connected graph of order $n$ with maximum degree $\Delta$ and minimum degree $\delta$. Let $\rho_{1} \leq \rho_{2} \leq \cdots \leq \rho_{n}$ are the eigenvalues of $A(G)$. Then for each $1 \leq$ $k \leq n$,

$$
\frac{\left|\rho_{n-k+1}\right|}{\Delta} \leq\left|1-\lambda_{k}\right| \leq \frac{\left|\rho_{n-k+1}\right|}{\delta} .
$$


Theorem 3.11 Let $G\left(G \neq K_{n}\right)$ be a connected graph of order $n$. Then $\lambda_{2}=1$ if and only if $G$ is a complete multipartite graph.

Proof By Lemma 3.10, if $\lambda_{2}=1$, then $\rho_{n-1}=0$, where $\rho_{n-1}$ is the second largest eigenvalue of $A(G)$. Hence the result follows from the fact that for any simple connected graph $G$ of order $n, \rho_{n-1} \leq 0$ if and only if $G$ is a complete multipartite graph [10]. On the other hand, when $G\left(G \neq K_{n}\right)$ is a complete multipartite graph, $\rho_{n-1}(G)=0$ [10]. This together with Lemma 3.10 imply that $\lambda_{2}=1$. The proof is completed.

Moreover, the following result on $\lambda_{n-1}$ is also obtained.

Theorem 3.12 Let $G$ be a connected graph of order $n$. Then $\lambda_{n-1} \geq 1$, the equality holds if and only if $G$ is a complete bipartite graph.

Proof Note that for any connected graph of order $n, \lambda_{1}=0$ and $\lambda_{n} \leq 2$. Since $\sum_{i=1}^{n} \lambda_{i}=n$, $\sum_{i=2}^{n-1} \lambda_{i} \geq n-2$ and hence $\lambda_{n-1} \geq 1$. Moreover, if $\lambda_{n-1}=1$, then $\lambda_{2}=\cdots=\lambda_{n-1}=1$ and $\lambda_{n}=2$ since $\sum_{i=2}^{n} \lambda_{i}=n$. This implies that $G$ is bipartite [2]. Moreover, since $\lambda_{2}=1$, combining with Theorem 3.11 we find that $G$ is complete bipartite graph. On the other hand, it is easy to check that if $G$ is a complete bipartite graph, then $\lambda_{n-1}=1$. This completes the proof.

Competing interests

The authors declare that they have no competing interests.

Authors' contributions

$J \mathrm{~L}$ carried out the proofs of the main results in the manuscript. J-MG and WCS participated in the design of the study and drafted the manuscript. All authors read and approved the final manuscript.

\section{Author details}

${ }^{1}$ School of Mathematics and Statistics, Minnan Normal University, Zhangzhou, Fujian, P.R. China. ${ }^{2}$ Center for Discrete Mathematics, Fuzhou University, Fuzhou, Fujian, P.R. China. ${ }^{3}$ Department of Mathematics, East China University of Science and Technology, Shanghai, P.R. China. ${ }^{4}$ Department of Mathematics, Hong Kong Baptist University, Kowloon Tong, Hong Kong, P.R. China.

\section{Acknowledgements}

The authors would like to thank the anonymous referees for their constructive corrections and valuable comments on this paper, which have considerably improved the presentation of this paper. This work was supported by NSF of China (Nos. 11101358, 61379021, 11371372); NSF of Fujian (No. 2014J01020); China Postdoctoral Science Foundation (No. 2014M551831); General Research Fund of Hong Kong; Faculty Research Grant of Hong Kong Baptist University.

Received: 6 January 2014 Accepted: 22 July 2014 Published: 21 Aug 2014

\section{References}

1. Cvetković, D, Rowlinson, P, Simić, S: An Introduction to the Theory of Graph Spectra. Cambridge University Press, Cambridge (2010)

2. Chung, F: Spectral Graph Theory. Am. Math. Soc., Providence (1997)

3. Chen, H, Jost, J: Minimum vertex covers and the spectrum of the normalized Laplacian on trees. Linear Algebra Appl. 437, 1089-1101 (2012)

4. Rojo, O, Soto, RL: A new upper bounds on the largest normalized Laplacian eigenvalue. Oper. Matrices 7, 323-332 (2013)

5. Banerjee, A: A few properties of the eigenvalues of normalized graph Laplacian (2012). arXiv:1210.5091v1 [math.CO]

6. Butler, S: Eigenvalues and structures of graphs. PhD dissertation, University of California, San Diego (2008)

7. Cavers, M: The normalized Laplacian matrix and general Randić index of graphs. PhD dissertation, University of Regina (2010)

8. Lu, M, Liu, H, Tian, F: An improved upper bound for the Laplacian spectral radius of graphs. Discrete Math. 309, 6318-6321 (2009)

9. Guo, J-M, Li, J, Shiu, WC: A note on the upper bounds for the Laplacian spectral radius of graphs. Linear Algebra Appl. 439, 1657-1661 (2013)

10. Cvetković, D, Doob, M, Sachs, H: Spectra of Graphs. Academic Press, New York (1980) 
10.1186/1029-242X-2014-316

Cite this article as: $L i$ et al.: Bounds on normalized Laplacian eigenvalues of graphs. Journal of Inequalities and Applications 2014, 2014:316

Submit your manuscript to a SpringerOpen ${ }^{\ominus}$ journal and benefit from:

- Convenient online submission

- Rigorous peer review

- Immediate publication on acceptance

Open access: articles freely available online

- High visibility within the field

- Retaining the copyright to your article

Submit your next manuscript at $\gg$ springeropen.com 